Arch. Tierz., Dummerstorf 46 (2003) 3, 273-276

Aus der Thüringer Landesanstalt für Landwirtschaft Jena, Abteilungen Tierproduktion ${ }^{1}$ und Untersuchungswesen ${ }^{2}$

SIMONE MÜLLER ${ }^{1}$, WERNER REICHARDT ${ }^{1}$, HORST HARTUNG ${ }^{2}$ und BÄRBEL ECKERT ${ }^{2}$

\title{
Analyse der Fettsäurenzusammensetzung des Rohfettes von Prüffut- ter für Schweine (Kurzmitteilung)
}

\author{
Herrn Professor Dr. Dr. h. c. Klaus Ender zum 60. Geburtstag gewidmet
}

\begin{abstract}
Title of the paper: Analysis of the fatty acid composition of the raw fat from the feed of pigs which are examined to her performance (short communication)

The raw fat of 14 examining feeds from 13 German performance testing centres for pigs was extracted 2001 and analysed for the fatty acid composition by means of gas chromatography. Besides a great variation of the raw fat content $(\mathrm{s} \%=42)$ was to state that with $14.4 \mathrm{~g} / \mathrm{kg}$ feed on average the content of polyunsaturated fatty acids (PUFA) was high. Examinations of the raw fat of wheat, rye and barley showed that the high proportions in linoleic and linolenic acid are brought in the fattening rations primarily by the cereal components. A limitation of the PUFA proportions below $15 \mathrm{~g} / \mathrm{kg}$ feed therefore doesn't seem to be practicable in the examining feed of performance testing centres for pigs. The additional variation in the fatty acid composition of examining feeds caused by added fats or oils should however be limited according to a better standardization.
\end{abstract}

Key Words: performance testing of pigs, examining feed, raw fat, fatty acid composition

\section{Zusammenfassung}

Das Rohfett von 14 Prüffutterproben aus 13 deutschen Leistungsprüfstationen für Schweine wurde 2001 extrahiert und gaschromatographisch auf die Fettsäurenzusammensetzung untersucht. Neben einer großen Variation des Rohfettgehaltes $(\mathrm{s} \%=42)$ war mit $14,4 \mathrm{~g} / \mathrm{kg}$ Futter ein im Mittel hoher Gehalt an Polyenfettsäuren (PUFA) festzustellen. Untersuchungen des Rohfettes von Weizen, Roggen und Gerste ergaben, dass die hohen Anteile an Linol- und Linolensäure an den Mastrationen vor allem durch die Getreidekomponenten eingebracht werden. Eine Begrenzung des PUFA-Anteils im Prüffutter von Leistungsprüfstationen für Schweine auf Werte unterhalb von $15 \mathrm{~g} / \mathrm{kg}$ Futter scheint daher nicht praktikabel zu sein. Die durch die Auffettung bedingte zusätzliche Variation in der Fettsäurenzusammensetzung sollte jedoch im Sinne einer besseren Standardisierung der Prüffutter eingeschränkt werden.

Schlüsselwörter: Leistungsprüfung beim Schwein, Prüffutter, Rohfett, Fettsäurenzusammensetzung

1. Einleitung

Infolge der zunehmenden Beachtung der Fettsäuren der in der menschlichen Nahrung enthaltenen Fette und Öle für den Gesundheitszustand der Bevölkerung interessiert sich auch die Tierproduktionsforschung seit etwa zehn Jahren verstärkt für das Fettsäurenmuster tierischer Fette. Die beim Schwein durch Zucht und Fütterung bewirkte Zunahme des Anteils mehrfach ungesättigter Fettsäuren in den Fettdepots bewegt sich dabei in einem Zielkonflikt zwischen Beschaffenheitsanforderungen und diätetischer Qualität. Mehrjährige Untersuchungen zur Zusammensetzung der Fettsäuren von Fett aus der äußeren Rückenspeckschicht bei Schweinen aus der Thüringer Leistungsprüf- 
anstalt (REICHARDT u.a., 2003) gaben Anlass, die Variation der Fettsäurenzusammensetzung des Rohfettes der in Deutschland eingesetzten Prüffutter zu untersuchen. Die deutschen Leistungsprüfanstalten für die Tierart Schwein beschlossen daher 2001, Prüffutterproben auf ihre Fettsäurenzusammensetzung untersuchen zu lassen. Die hierzu und bei der Untersuchung von Getreide erzielten Ergebnisse sollen nachfolgend kurz vorgestellt werden.

\section{2.}

Material und Methoden

Die Extraktion der Fette aus den gemahlenen Prüffuttermischungen erfolgte im Originalzustand, während die Getreidekörner zuvor mittels Homogenisator zerkleinert wurden. $10 \mathrm{~g}$ Einwaage an Prüffutter oder Getreide sind ohne vorherigen Säureaufschluss mit $3 \mathrm{~g}$ wasserfreiem Natriumsulfat vermischt und mit $50 \mathrm{ml}$-Hexan mit einem ULTRA-TURRAX T 25 bei $13.500 \mathrm{U} / \mathrm{min}$. homogenisiert worden. $10 \mathrm{ml}$ eines Filtrat sind in einem 20 ml-Zentrifugenglas mit NS 14,5 mit 0,6 ml eines 30 \%igen Lösung von Natriummethylat in Methanol versetzt und 30 Sekunden geschüttelt worden. Nach der Zugabe von einer Spatelspitze von getrocknetem Calziumchlorid wurde nochmals 30 Sekunden geschüttelt und die Lösung zur Phasentrennung dann 3 bis 4 Stunden stehengelassen. Die Analyse der Fettsäurenzusammensetzung erfolgte mittels Kapillargaschromatographie $(1 \mu$ Injektionsvolumen aus dem Überstand mit einem Gaschromatographen des Typs VARIAN STAR 3400 CX und automatischer Probenaufgabe (VARIAN-CHROMPACK-Autosampler 8200). Die Angaben zu den Fettsäurenanteilen beziehen sich auf Masse-\% Fettsäurenmethylester. Fettsäuren tragen etwa zu $90 \%$ zur Fettmasse bei, was bei der Berechnung des Anteils polyungesättigter Fettsäuren (PUFA) im Futter zu berücksichtigen ist.

\section{Ergebnisse und Diskussion}

Tabelle 1 fasst die Ergebnisse zusammen, die bei der Analyse der Fettsäurenzusammensetzung des Rohfettes von 14 verschiedenen Prüffutterproben aus 13 deutschen Leistungsprüfanstalten (LPA) für die Tierart Schwein erhalten wurden. Die Prüffutterzusammensetzung entsprach nach den im Auftrag der LPA regional vorgenommenen Futtermittelanalysen in den Gehaltswerten den Vorgaben des Arbeitsausschusses für Leistungsprüfung in der Tierzucht (ALZ). Acht Prüffutter enthielten laut Deklaration Fett- oder Ölzusätze, zwei waren ohne solche, bei vier Proben fehlten die Angaben. Der mittlere PUFA-Gehalt der untersuchten Prüffutter lag bei $14,4 \mathrm{~g} / \mathrm{kg}$ Originalsubstanz. Seine hohe Variationbreite $(8,7$ bis $27,0 \mathrm{~g}$ PUFA $/ \mathrm{kg})$ ist vorwiegend auf die Variation des Rohfettgehaltes zurückzuführen. Sollen $12 \%$ PUFA im Rückenfett nicht überschritten werden (Schweizer Grenzwert für eine gute Fettqualität im Rückenspeck) darf nach SCHULZ und ALERT (2002) der PUFA-Gehalt im Mastfutter nur 10 - $16 \mathrm{~g} / \mathrm{kg}$ betragen. PERDRIX und STOLL (1995) gaben eine obere Grenze von 0,8 g Polyensäuren / MJ verdauliche Energie beim Schwein (ca. $12 \mathrm{~g}$ PUFA/kg Futter) für die Sicherung einer guten Qualität des Rückenspecks von Schweinen vor. Sieben Prüffutter übertrafen einen Grenzwert von $12 \mathrm{~g}$ PUFA $/ \mathrm{kg}$, wofür besonders Linolsäureanteile von über $50 \%$ verantwortlich sind. Bei der Suche nach möglichen Ursachen sind als übliche Rationsbestandteile von Schweineprüffutter einige Getreide- und zwei Sojaproben auf die Fettsäurenzusammensetzung ihrer Rohfette untersucht worden (Tab. 2). Die Ergebnisse stimmen gut mit Daten von BURGSTALLER u.a. (1993) überein. 
Sie verdeutlichen, dass bereits die Getreidekomponenten des Prüffutters mit hohen Anteilen an Linol- und Linolensäure im Rohfett wesentlich zum PUFA-Gehalt der Ration beitragen und nur zu einem geringeren Teil die Auffettung der Pellets oder die Sojabestandteile. Bereits GLÄSER (2000) verwies darauf, dass die Getreiderationen ohne Fettzulage einen Wert von $12 \mathrm{~g}$ PUFA/kg überschreiten können. Eine typische Futtermischung, die $50 \%$ Gerste, $35 \%$ Weizen und $15 \%$ Sojaextraktionsschrot enthält, würde nach den Daten von Tabelle 2 ohne Auffettung eine PUFA-Konzentration von $11,1 \mathrm{~g} / \mathrm{kg}$ aufweisen. Die Spannweite von diesem Wert bis 12 oder $15 \mathrm{~g}$ PUFA/kg

\section{Tabelle 1}

Ergebnisse der Fettsäurenanalyse bei Schweinefutter $(n=13$ Leistungsprüfanstalten mit $n=14$ verschiedenen Futterproben) (Results of the fatty acid analysis at pork feed $(n=13$ performance testing centres with $n=14$ different feed tests)

\begin{tabular}{|c|c|c|c|c|c|}
\hline Merkmal & Name der Fettsäure & $\varnothing$ & $\mathrm{s}$ & Min & $\operatorname{Max}$ \\
\hline$\% \mathrm{C} 12: 0$ & Laurinsäure & 0,84 & 1,02 & 0,02 & 2,91 \\
\hline$\%$ C14:0 & Myristinsäure & 0,44 & 0,35 & 0,10 & 1,08 \\
\hline$\% \mathrm{C} 14: 1$ & Myristoleinsäure & 0,04 & 0,08 & 0 & 0,29 \\
\hline$\%$ C15:0 & Pentadecansäure & 0,06 & 0,02 & 0,03 & 0,08 \\
\hline$\%$ C16:0 & Palmitinsäure & 15,17 & 1,70 & 12,52 & 17,98 \\
\hline$\% \mathrm{C} 16: 1$ & Palmitoleinsäure & 0,20 & 0,08 & 0,12 & 0,39 \\
\hline$\%$ C17:0 & Margarinsäure & 0,10 & 0,02 & 0,07 & 0,15 \\
\hline$\%$ C $17: 1$ & Heptadecensäure & 0,04 & 0,02 & 0 & 0,06 \\
\hline$\%$ C18:0 & Stearinsäure & 2,23 & 0,59 & 1,37 & 3,53 \\
\hline$\%$ C18:1 & Ölsäure & 19,55 & 2,72 & 16,32 & 24,44 \\
\hline$\% \mathrm{C} 18: 2$ & Linolsäure & 51,09 & 3,31 & 44,63 & 55,52 \\
\hline$\% \alpha-C 18: 3$ & Alpha-Linolensäure & 6,18 & 0,77 & 4,78 & 7,89 \\
\hline$\% \gamma-\mathrm{C} 18: 3$ & Gamma-Linolensäure & 1,02 & 0,18 & 0,61 & 1,25 \\
\hline$\%$ C20:0 & Arachinsäure & 0,24 & 0,04 & 0,15 & 0,29 \\
\hline$\%$ C20:1 & Zikosaensäure & 0,56 & 0,11 & 0,34 & 0,74 \\
\hline$\%$ C20:2 & Zikosadiensäure & 0,07 & 0,02 & 0,02 & 0,09 \\
\hline$\%$ C20:3 & Zikosatriensäure & 0,01 & 0,03 & 0 & 0,10 \\
\hline$\%$ C20:5 & Eicosapentaensäure & 0,001 & 0,004 & 0 & 0,01 \\
\hline$\%$ C21:0 & Heneicosansäure & 0,007 & 0,009 & 0 & 0,02 \\
\hline$\%$ C22:0 & Behensäure & 0,22 & 0,07 & 0,11 & 0,37 \\
\hline$\%$ C22:1 & Erucasäure & 0,22 & 0,17 & 0,04 & 0,74 \\
\hline$\%$ C22:2 & Docosadiensäure & 0,12 & 0,05 & 0,05 & 0,22 \\
\hline$\%$ C23:0 & Tricosansäure & 0,15 & 0,03 & 0,09 & 0,20 \\
\hline$\%$ C24:0 & Lignocerinsäure & 0,11 & 0,03 & 0,06 & 0,16 \\
\hline$\%$ C24:1 & Nervonsäure & 0,13 & 0,07 & 0,04 & 0,28 \\
\hline Merkmal & Erläuterung & $\varnothing$ & $\mathrm{s}$ & Min & Max \\
\hline \% Rohfett & g Rohfett $/ \mathrm{kg}=\%$ Rohfett $* 10$ & 2,77 & 1,16 & 1,58 & 5,78 \\
\hline$\%$ PUFA & Summe aller polyungesättigte Fettsäuren & 58,50 & 3,41 & 51,90 & 62,97 \\
\hline g PUFA/kg & $=\mathrm{g}$ Rohfett $/ \mathrm{kg} * 0,9 * \%$ PUFA $/ 100 \%$ & 14,38 & 5,51 & 8,74 & 27,00 \\
\hline
\end{tabular}

$\varnothing=$ Mittelwert; $\mathrm{s}=$ Standardabweichung; Min = Minimum; Max = Maximum

Tabelle 2

Ausgewählte Ergebnisse der Fettsäurenanalyse des im Getreide enthaltenen Rohfettes (Selected results of the fatty acid analysis of the raw fat contained in cereals)

\begin{tabular}{|c|c|c|c|c|c|c|c|c|c|c|c|}
\hline Merkmal & & \multicolumn{2}{|c|}{$\mathrm{C} 18: 2 \%$} & \multicolumn{2}{|c|}{$\alpha-\mathrm{C} 18: 3 \%$} & \multicolumn{2}{|c|}{$\gamma-\mathrm{C} 18: 3 \%$} & \multicolumn{2}{|c|}{ PUFA $^{3)} \%$} & \multirow{2}{*}{$\begin{array}{l}\left.\text { Fett }^{4}\right) \\
\mathrm{g} / \mathrm{kg}\end{array}$} & \multirow{2}{*}{$\begin{array}{l}\text { PUFA } \\
\mathrm{g} / \mathrm{kg}\end{array}$} \\
\hline Probenart & $\mathrm{n}$ & $\varnothing$ & $\mathrm{S}$ & $\varnothing$ & $\mathrm{S}$ & $\varnothing$ & $\mathrm{s}$ & $\varnothing$ & $\mathrm{s}$ & & \\
\hline $\begin{array}{l}\text { Weizen }{ }^{1)} \\
\text { Roggen }{ }^{1)} \\
\text { Gerste }^{2)}\end{array}$ & $\begin{array}{l}20 \\
10 \\
7 \\
\end{array}$ & $\begin{array}{l}60,03 \\
57,06 \\
54,41 \\
\end{array}$ & $\begin{array}{l}1,26 \\
0,89 \\
0,96 \\
\end{array}$ & $\begin{array}{l}4,54 \\
8,71 \\
6,56 \\
\end{array}$ & $\begin{array}{l}0,45 \\
0,38 \\
1,20 \\
\end{array}$ & $\begin{array}{l}0,82 \\
0,85 \\
0,77 \\
\end{array}$ & $\begin{array}{l}0,07 \\
0,17 \\
0,02 \\
\end{array}$ & $\begin{array}{l}65,59 \\
66,90 \\
62,00 \\
\end{array}$ & $\begin{array}{l}1,51 \\
1,00 \\
1,07 \\
\end{array}$ & $\begin{array}{l}19 \\
16 \\
22 \\
\end{array}$ & $\begin{array}{l}11,2 \\
9,6 \\
12,3 \\
\end{array}$ \\
\hline $\begin{array}{l}\text { Sojaschrot } \\
\text { Sojaextrak- } \\
\text { tionsschrot }\end{array}$ & $\begin{array}{l}1 \\
1\end{array}$ & $\begin{array}{l}51,21 \\
53,71\end{array}$ & - & $\begin{array}{l}7,43 \\
6,87\end{array}$ & - & $\begin{array}{l}1,08 \\
1,45\end{array}$ & - & $\begin{array}{l}59,85 \\
62,08\end{array}$ & - & $\begin{array}{l}177 \\
12\end{array}$ & $\begin{array}{l}95,3 \\
6,7\end{array}$ \\
\hline
\end{tabular}

1) Winter; ${ }^{2)} 3 \mathrm{x}$ Winter, 4 x Sommer; ${ }^{3)}$ weitere gefundene PUFA: C20:2, C20:3, C20:5 und C22:2;

mittlerer Gehalt in der Originalsubstanz nach Angaben der Deutsch Landwirtschafts-Gesellschaft 
ist für die aus energetischen, ökonomischen und technologischen Gründen notwendige Fettzulage zu gering, so dass Überschreitungen solcher PUFA-Grenzwerte im Prüffutter zwangsläufig auftreten.

\section{Schlussfolgerungen}

Bei einer ALZ-gerechten Zusammensetzung des LPA-Prüffutters sind Grenzwerte von 1,2 oder 1,5\% für den PUFA-Gehalt, die eine verarbeitungstechnologische Eignung des Schweinerückenspecks zu Dauerwaren und eine ausreichende oxidative Haltbarkeit, sensorische Qualität und Konsistenz seiner Verarbeitungsprodukte gewährleisten sollen, bereits durch die Hauptkomponenten der praktischen Mischfutterrationen kaum zu gewährleisten. Eine Futterauffettung bewirkt schnell eine Überschreitung dieser Grenzwerte. Sie sollte daher nach Art des Fettes und nach Menge in Zukunft standardisiert erfolgen. Eine fakultative Analytik der Fettsäurenzusammensetzung von LPAPrüffutter ist sinnvoll bei gewünschter gezielter Einflussnahme auf die Fettqualität der Mastprodukte.

\section{Literatur}

BURGSTALLER, G.; JATSCH, C.; BEESTEN, L.:

Zur Fettqualität bei schweren Mastschweinen in Abhängigkeit vom Verabreichungszeitraum gleicher Rapsölmengen. Züchtungskunde 65 (1993) 4, 306-317

GLÄSER, K.R.:

Untersuchungen zur Eignung der Fettzahl als Kriterium für die Einschätzung der Fettqualität von Mastschweinen unter dem Einfluss der Fütterung und im Hinblick auf die Qualität von Verarbeitungsprodukten. ETH Zürich, Diss., 2000

PERDRIX, M.-F.; STOLL, P.:

Wie beeinflußt das Futter die Fettzahl der Schweine ? AgrarForschung 2 (1995) 1, 21-24

REICHARDT, W.; GERNAND, E.; MÜLLER, S.; HARTUNG, H.; ECKERT, B.:

Erhebungen zur Fettsäurenzusammensetzung von Rückenfett bei Thüringer Schweinen sowie zum Fett von Thüringer Knackwürsten aus dem Einzelhandel. Arch. Tierz., Dummerstorf 46 (2003) 3, 257-267

SCHULZ, E.; ALERT, H.-J.:

Beiträge zur Fütterung von Schweinen mit hoher Leistung. Internationaler Kongress „Wirtschaftliche Schweineproduktion unter neuen Rahmenbedingungen“" der Sächsischen Landesanstalt für Landwirtschaft, Leipzig, 28.02.-02.03.2002, Tagungsband, 59-71

Eingegangen:16.12.2002

Akzeptiert: 06.05.2003

Anschrift der Verfasser

Dr. SIMONE MÜLLER, Dr. WERNER REICHARDT, Dr. HORST HARTUNG,

BÄRBEL ECKERT

Thüringer Landesanstalt für Landwirtschaft

Am Rennsteig 3

D-99819 Oberellen - OT Clausberg 